\title{
Wissenschaftliche Pluralität meint Pluralität der Erkenntnisformen
}

\author{
In Zeiten erheblichen gesellschaftlichen Wandels \\ braucht es an Hochschulen mehr, als existie- \\ rendes Wissen zu vermitteln. Studierende sind \\ zur Reflexion und Gestaltung der Gewinnung \\ von Wissen, das heißt von Erkenntnisprozessen \\ selbst, zu befähigen, damit sie in der Welt \\ von morgen die richtigen Fragen stellen können. \\ Welche Pluralität von Erkenntnisformen spielt \\ eine Rolle und vor welchen Herausforderungen \\ steht die Wissenschaftslandschaft? \\ Von Silja Graupe und Harald Schwaetzer
}

$\mathbf{S}$ chneidewind und Singer-Brodowski messen Hochschulen als „institutionalisierten Reflexionsorten“ in modernen Gesellschaften eine besondere Bedeutung zu, gerade auch im Hinblick auf die Herausforderungen im Zusammenhang mit nachhaltigen Entwicklungsprozessen (2014). Statt lediglich weiterer funktionaler Ausdifferenzierungen benötige die Gesellschaft auch Formen der Reflexion und Integration in besonderem Maße. Als „Brückenbauer zwischen den Systemen“ (ebd.) könnten dabei gerade Hochschulen wesentliche Reflexions- und Transformationsaufgaben für die Gesellschaft erfüllen. Wie Schneidewind und Singer-Brodowski betonen, ist hier nicht nur die Forschung, sondern auch die Lehre angesprochen (ebd.). Wie aber können junge Menschen dazu befähigt werden, gesellschaftliche Entwicklungen so zu reflektieren, dass sie an verantwortlicher schöpferischer Gestaltung aktiv teilhaben können? In diesem Beitrag diskutieren wir dies kurz in erkenntnistheoretischer Hinsicht und markieren vor diesem Hintergrund einige institutionelle Herausforderungen. Dabei nehmen wir besonderen Bezug auf die Lage der ökonomischen Bildung.

\section{Ökonomische Bildung und Pluralität}

Gegenwärtig wird in der ökonomischen Bildung vielfach der Ruf nach Pluralität erhoben. Für uns geht es dabei nicht allein um eine Pluralität der Erkenntnisgegenstände, sondern auch um eine reflektierte Pluralität von Erkenntnisprozessen unter Wahrung eines Wahrheitsanspruches (Anders 1993). Hierzu ist wiederum nicht allein eine Theorienvielfalt vonnöten (auch wenn dies bereits vielfach einen Fortschritt in der ökonomischen Bildung darstellte). Weit zentraler erscheint uns, Studierende zum Umgang mit grundsätzlich unterschiedlichen Erkenntnisformen zu befähigen. Hierbei sind zumindest fünf Formen zu unterscheiden (siehe Abbildung 1), die sich wechselseitig bedingen, nicht aber ersetzen:

\section{Beeinflusste Erkenntnis}

Polanyi (1977) schreibt, dass der hauptsächliche Einfluss der Wissenschaft auf den modernen Menschen nicht durch den Fortschritt der Technologie ausgeübt würde, sondern durch die „imaginären Effekte“ der Wissenschaft auf unsere Weltanschauung. Diese imaginären Effekte wirken allerdings zumeist nicht auf der Ebene des Bewusstseins, sondern bleiben überwiegend unbewusst. In der Sprache der Kognitionsforschung kann Wissenschaft gedankliche Deutungsrahmen, Frames genannt, prägen, die ihrerseits beeinflussen, was von Einzelpersonen oder Gesellschaften überhaupt als relevante Fakten und Informationen wahrgenommen und verarbeitet werden kann.

Eine neue Studie (Graupe 2017) zeigt am Beispiel von Paul Samuelsons Economics und Mankiws Economics, zwei der weltweit wichtigsten wirtschaftswissenschaftlichen Standardlehrbücher, wie die ökonomische Bildung gerade auf dieser vorreflexiven Ebene die Meinungsbildung und damit die grundlegenden Denk- und Wahrnehmungsweisen umgestal-

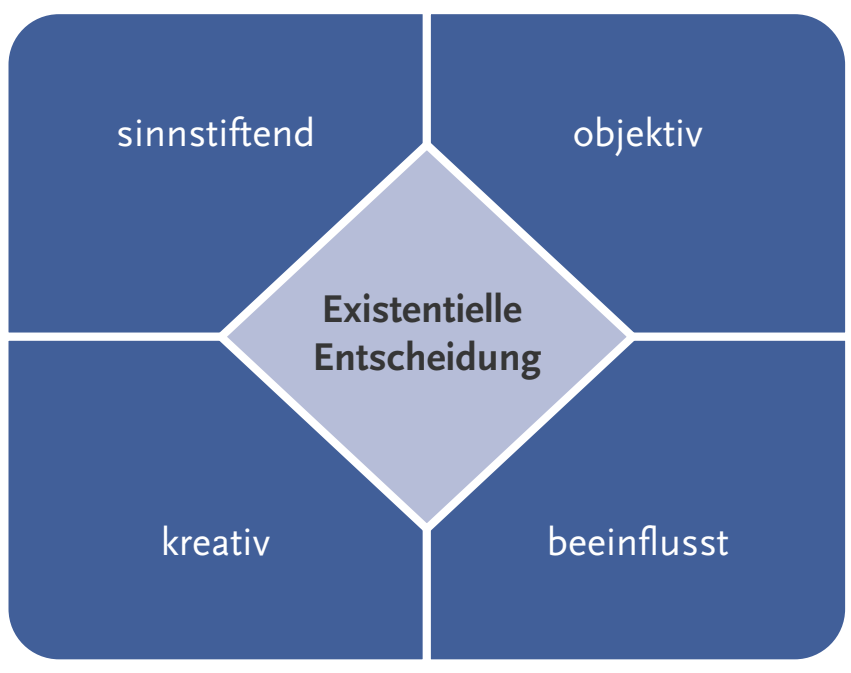

Abbildung 1: Formen von Erkenntnisprozessen

(eigene Darstellung) 
ten kann. Dies geschieht dabei bis zur Ebene der „Deep Seated Frames“, „die unser generelles Verständnis von der Welt strukturieren, unsere Annahmen von der Welt zum Beispiel aufgrund unserer moralischen und politischen Prinzipien, die für uns schlicht, wahr' sind - die also unseren eigenen Common Sense ausmachen“ (Lakoff/Wehling 2016). Problematisch wird solche Beeinflussung vor allem dann, wenn sie manipulativ ist, das heißt für Studierende tatsächlich unsichtbar verläuft und keinen offenen Raum der Aneignung eröffnet, sondern gezielt persönlichkeitsverändernde Prozesse in Gang setzt, die sich unterhalb des wachen Bewusstseins und seines kritischen Vermögens abspielen sollen.

\section{Objektive Erkenntnis}

Auch hier ist stets schon entschieden, welche vorgegebenen Erkenntniswege Studierende zu verfolgen haben, aber zumindest sind diese Wege bewusst zu beschreiten. Ziel- und Fluchtpunkt ist dabei, kurz gesagt, jenseits aller Erfahrung rein logisch zu argumentieren und insbesondere abstrakt zu rechnen. Jeder Erfahrungsbezug ist dem Primat formaler Strukturen zu opfern. Denn objektiv zu denken heißt, sich bewusst von subjektiven Bezügen (der Genese) zu distanzieren und einen „Blick von nirgendwo“ (Thomas Nagel) (als Blick der Geltung) einzunehmen, das heißt, sich mit kühlem Gleichmut Bilder von der Welt zu machen, die allerdings im Extremfall reine Scheinwelten darstellen können. In gegenwärtigen Diskussionen wird die Realitätsferne der ökonomischen Theorie oft kritisiert.

Doch im Reich der Objektivität stellt diese Ferne keinen Unfall dar, sondern ist eine bewusst zu vollbringende Verstandesleistung. Diese Leistung hilft, sich vom alltäglichen Common Sense und seinen „Deep Seated Frames“ zu distanzieren. Zudem erlaubt sie es, sich über Voraussetzungen und Grenzen abstrakter Theorien und Modelle aufzuklären (ein Reflexionswissen, das etwa in der letzten Finanzkrise systematisch im Hinblick auf die auf Optionsmärkten zur Anwendung kommenden finanzmathematischen Modelle fehlte). Jenes oft stillschweigende Wissen vermag aber auch hier nicht in den Blick zu geraten.

Unsere Sorge ist, dass sich die ökonomische Standardbildung gegenwärtig überwiegend in den in der Abbildung dargestellten rechten Quadranten vollzieht: Studierende werden durch das Erlernen mathematisch-objektiver Modelle einerseits zur Distanzierung von der Welt angeleitet (Objektivität) und andererseits zu unwillkürlichen Reaktionen im Denken und Handeln auf der Basis von Weltanschauungen verleitet, die ihnen selbst nicht reflexiv zugänglich sind (Beeinflussung). Weltferne und Indoktrination drohen sich so verbinden zu können, insbesondere wenn die Beeinflussung manipulativ erfolgt. Wir plädieren vor diesem Hintergrund dafür, die (wirtschafts)wissenschaftliche Bildung wesentlich um die Befähigung zur sinnstiftenden und kreativen Erkenntnis zu erweitern.

\section{Sinnstiftende Erkenntnis}

„To talk about sensemaking is to talk about reality as an ongoing accomplishment that takes form when people make retroperspective sense of the situations in which they find themselves and their creations. There is a strong reflexive quality to these processes. People make sense of things by seeing a world on which they already imposed what they believe" (Weick 1995).

Beeinflusste Erkenntnis kennt schnelle, automatisch-unbewusste Reaktionen auf Vorstellungsbilder, objektive Erkenntnis lediglich den bewussten Nachvollzug formal vorgegebener Verstandesoperationen. Sinnstiftende Erkenntnis richtet sich nun unmittelbar auf die Prozesse der Entstehung von Vorstellungen, Konzepten und Bildern, durch die sich Weltanschauung (im doppelten Sinne) vollzieht. Vom Bewusstsein begleitetes Denken setzt hier ein, bevor ein konkreter Sinn gestiftet ist, weil es im Erkenntnisvollzug selbst aktiv tätig wird. „A focus on sensemaking induces a mindset to focus on process" (Weick 1995).

Sinnstiftende Erkenntnis meint selbstreflexive, dynamische Erkenntnisprozesse, in denen (anders als aus der sicheren Distanz der Objektivität) einerseits gedankliche Deutungsrahmen in ihrer konstitutiven Bedeutung für Welt und Mensch, andererseits Welt (etwa in einer phänomenologischen Naturphilosophie) in Erscheinung treten. Soll Bildung zu Sinnstiftung befähigen, muss der Entstehungsprozess der Sinnstiftung selbst zum Reflexionsort werden. So kann manipulativer Beeinflussung entgegengewirkt und Beeinflussung selbst reflektiert werden. Hierfür bedarf es etwa der Schulung eines umfassenden Geschichtsbewusstseins, um denkbar werden zu lassen, wie wir aus der Vergangenheit herkommend in der Gegenwart sein können.

\section{Kreative Erkenntnis}

Hier geht es um die schöpferisch-dynamische Seite des Erkenntnisvollzugs, der sich auf die Zukunft ausrichtet. Wie können wir erkennen, wie wir in Zukunft denken wollen? Wie schaffen wir eine prinzipielle Offenheit des Erkennens für das Neue und Überraschende, für das Noch-Nicht-Seiende, aber Sein-Sollende? Kreative Erkenntnis ist wesentlich eine fragende Haltung: Wie können wir heute lernen und lehren, morgen die richtigen Fragen stellen zu können? Um sich solchen Fragen stellen zu können, bedarf es auch moralischer Entschlossenheit.

\section{Existentielle Entscheidung}

Aus unserer Sicht gibt die heutige Bildung an Hochschulen der Sinnstiftung und Kreativität auch und gerade in den Wirtschaftswissenschaften zu wenig Raum. Sollen Hochschulen aber tatsächlich zu „Reflexionsorten“ werden, die transformativ in der Gesellschaft wirken, so müssen sie nicht nur diese 
beiden Felder transformativ-reflexiver Bildung in die Lehre integrieren. Es geht um noch mehr: Darum, Studierende zu befähigen, sich von Situation zu Situation für die eine oder andere Erkenntnisform entscheiden zu können. Dies aber ist keine rationale Wahl, weil weder ein gegebener Möglichkeitsraum noch gegebene innere Entscheidungsregeln bestehen. Es ist eine „existentielle Entscheidung“ (Barth 2016), da sie immer auch die Frage betrifft, wie Studierende leben und sich zur Welt und sich selbst verhalten wollen. Die Fähigkeit zu einer solchen Art der Entscheidung zu schulen, ist auch und gerade Aufgabe der Bildung.

\section{Institutionelle Herausforderungen}

Uns ist bewusst, dass dies die gegenwärtige (ökonomische) Bildung an Hochschulen vor Herausforderungen stellt. Schneidewind und Singer-Brodowski schlagen vor, „,Inseln der Heterodoxie im deutschen Wissenschaftssystem zu kultivieren“ (2014). Wir haben die Cusanus Hochschule mitgegründet und entwickeln sie kontinuierlich fort, um eine solche „Insel“ zu schaffen, die Studierende in Ökonomie und Philosophie gleichsam zur Exzellenz in der existentiellen Entscheidung im vorgenannten Sinne befähigt (Graupe/Schwaetzer 2015). Diese und weitere „Inseln“ gilt es zunächst institutionell wie finanziell zu stärken, wobei nicht nur an Hochschulen, Institute oder Studiengänge, sondern etwa auch an Studierendeninitiativen (wie das Netzwerk Plurale Ökonomik) zu denken ist. Zugleich ist ein Netz solcher „Inseln“ zu bilden und zu kultivieren - auch und gerade über die Grenzen der Hochschulen hinaus. Dabei kann für Hochschulen die Zusammenarbeit mit außeruniversitären Forschungseinrichtungen eine besondere Rolle spielen, um Studierende in transdisziplinäre Lehr- und Forschungskontexte einzubinden. Fernziel ist, die so neu entstandenen Bildungsformen in der Hochschul- landschaft insgesamt fruchtbar zu machen und so, um in der Metapher zu bleiben, tatsächlich Bildungsneuland zu gewinnen.

\section{Literatur}

Anders, G. (1993): Mensch ohne Welt. Schriften zur Literatur und Kunst. München, Beck.

Barth, H. (2016): Grundriß einer Philosophie der Existenz. Herausgegeben von Christian Graf, Cornelia Müller und Harald Schwaetzer. Regensburg, Roderer.

Graupe, S. (2017): Beeinflussung und Manipulation in der ökonomischen Bildung. Düsseldorf, FGW (im Erscheinen).

Graupe, S./Schwaetzer, H. (Hrsg.) (2015): Bildung gestalten. Akademische Aufgaben der Gegenwart. Coincidentia Beiheft 5. Münster, Aschendorff.

Lakoff, G./Wehling, E. (2016): Auf leisen Sohlen ins Gehirn. Politische Sprache und ihre heimliche Macht. Heidelberg, Carl-Auer.

Polanyi, M./Prosch, H. (1977): Meaning. Chicago/London. University of Chicago Press.

Schneidewind, U./Singer-Brodowski, M. (2014): Transformative Wissenschaft. Marburg, Metropolis.

Weick, K. E. (1995): Sensemaking in Organizations. Thousand Oaks, Sage Publications.

\section{AUTOREN + KONTAKT}

Dr. Silja Graupe ist Vizepräsidentin der Cusanus Hochschule und Professorin für Ökonomie und Philosophie.

Dr. Harald Schwaetzer ist Vizepräsident der Cusanus Hochschule und Professor für Philosophie.

Cusanus Hochschule, Mandatstr. 1, 54470 Bernkastel-Kues. Tel.: +49 6531-972425-7, E-Mail: silja.graupe@cusanus-hochschule.de, harald.schwaetzer@cusanus-hochschule.de

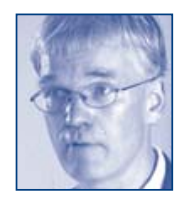

Nachhaltigkeit

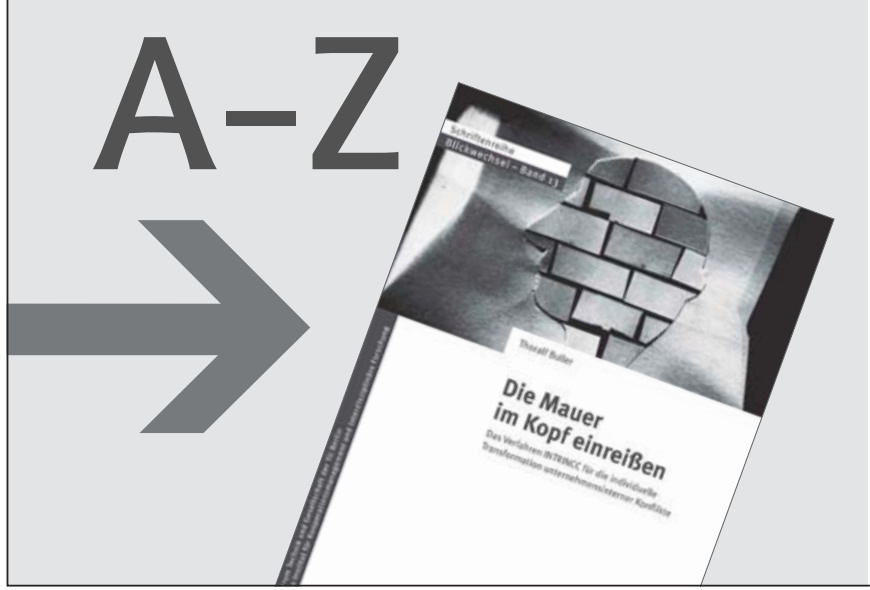

\section{B wie Betriebsklima}

Begegnungen mit anderen Menschen machen unseren Alltag aus, vielen davon können wir beruflich bedingt nicht ausweichen. Führen diese Kontakte zu Konflikten, so leiden die Betroffenen, aber auch die Arbeitsatmosphäre und letztlich die Effizienz des Unternehmens. Im INTRINCC-Verfahren werden Innere Sprache und Selbstbericht verbunden und Wege aufgezeigt, den Kern eines Konflikts individuell zu deuten und letztlich zu versachlichen.

\section{T. Buller}

Die Mauer im Kopf einreißen

Das Verfahren INTRINCC für die individuelle Transformation unternehmensinterner Konflikte

192 Seiten, broschiert, 29,95 Euro, ISBN 978-3-96006-006-2

Erhältlich im Buchhandel oder versandkostenfrei

innerhalb Deutschlands bestellbar unter www.oekom.de

Die guten Seiten der Zukunft 